\title{
Quantitative Analysis and Characteristics of the Electrograms Recorded Within the Non-Coronary Aortic Sinus of Valsalva
}

\author{
Shigeki Hiramatsu, MD**; Hiroshi Tada, MD*; Yuichiro Sakamoto, MD ${ }^{\dagger}$; \\ Kenichi Kaseno, MD; Chizuru Sato, MD; Tadanobu Irie, MD; Miki Yokokawa, MD; \\ Satoshi Nagase, MD**; Shigeto Naito, MD; Kengo Fukushima Kusano, MD**; \\ Masakazu Yamagishi, MD ${ }^{\dagger}$; Tohru Ohe, MD**; Kazutaka Aonuma, MD*; \\ Shigeru Oshima, MD; Koichi Taniguchi, MD
}

\begin{abstract}
Background: Some supraventricular tachycardias could be ablated from the non-coronary sinus of Valsalva (NSV). However, the characteristics of the NSV electrograms have not been clarified.

Methods and Results: A quantitative analysis of the NSV electrograms was performed in 5 patients with tachycardias arising from near the atrioventricular node (AVN) and the His-bundle region, and in 20 control subjects. In another 7 control subjects, the NSV electrograms were compared with those recorded at the left and right sinus of Valsalva (LSV and RSV). The NSV electrograms during sinus rhythm had a larger atrial amplitude than ventricular amplitude, and the ratio of the atrial amplitude to the ventricular amplitude was usually $>1$, which was apparently different from the LSV and RSV electrograms. A tiny but distinct His-bundle deflection was sometimes recorded at the NSV during sinus rhythm while it was not during the tachycardia. The distance to the His-bundle region in the anteroseptal right atrium was shorter from the NSV than from the RSV or LSV.

Conclusions: The precise identification of the catheter position at the NSV is possible using the characteristics of the electrograms. Much attention should be paid during ablation to the NSV because of its vicinity to the AVN and His-bundle region. (Circ J 2009; 73: 838-845)
\end{abstract}

Key Words: Catheter ablation; Electrogram; His-bundle region; Non-coronary aortic sinus of Valsalva; Tachycardia

$\mathbf{R}$ ecent studies have demonstrated that some supraventricular tachycardias that have their origins near the atrioventricular node (AVN) or His-bundle region can be eliminated by radiofrequency (RF) catheter ablation from the non-coronary sinus of Valsalva (NSV)!-6 We have also reported 2 cases of supraventricular tachycardias, which consisted of an atrial tachycardia (AT) ${ }^{1}$ and atrioventricular (AV) orthodromic tachycardia (AVRT)? However, the characteristics of the electrograms recorded from the NSV during normal sinus rhythm as well as during the tachycardia have not been sufficiently examined. Furthermore, the difference between the electrograms recorded from the NSV and those recorded from the other 2 aortic sinuses of Valsalva has not been clarified.

Even if an early atrial activation during the tachycardia is found on the right atrial (RA) anteroseptum, sufficient

(Received September 16, 2008; revised manuscript received December 5, 2008; accepted January 4, 2009; released online March 31, 2009) Division of Cardiology, Gunma Prefectural Cardiovascular Center, Maebashi, *Division of Cardiology, Tsukuba University Hospital, Graduate School of Comprehensive Human Sciences, University of Tsukuba, Tsukuba, **Department of Cardiovascular Medicine, Okayama University Graduated School of Medicine, Density and Pharmaceutical Science, Okayama and Division of Cardiovascular Medicine, Kanazawa University Graduate School of Medical Science, Kanazawa, Japan

Mailing address: Hiroshi Tada, MD, Division of Cardiology, Tsukuba University Hospital, Graduate School of Comprehensive Human Sciences, University of Tsukuba, 1-1-1 Tennodai, Tsukuba 305-8575, Japan. E-mail: htada@md.tsukuba.ac.jp

All rights are reserved to the Japanese Circulation Society. For permis-

sions, please e-mail: cj@j-circ.or.jp deliveries of the RF energy at that site might be impossible because of the high risk for the impairment of the AV conduction!-6 In such a situation, RF ablation at the NSV might eliminate the tachycardia with no complications ${ }^{1-6}$ However, because of the vicinity to the AVN and His-bundle region, the RF catheter ablation at the NSV always carries the risk of impairing the conduction of the AVN. In fact, the first case report of RF ablation from the NSV was a case in which a complete AV block was created by the RF ablation from the NSV? However, the detailed information on the distance from the NSV to the AVN or His-bundle region or the prevalence of recording a His-bundle potential at the NSV, both of which seem to be very important to estimate the risk of AVN conduction block during the RF energy application from the NSV, 8 was lacking. Accordingly, in the present study, we performed a quantitative measurement of the electrograms recorded from the aortic sinus of Valsalva during sinus rhythm and/or the tachycardia to clarify those points.

\section{Methods}

\section{Study Population and Protocol}

This study consisted of 3 protocols as follows.

Protocol I: Recording of the NSV Electrograms in Patients With Supraventricular Tachycardia The mapping and recording of the NSV electrograms were performed during sinus rhythm as well as during the tachycardia in 5 patients with paroxysmal supraventricular tachycardia (3 women; mean age, $64 \pm 12$ years) in whom successful elimination of the tachycardia could be achieved by an RF energy 
A

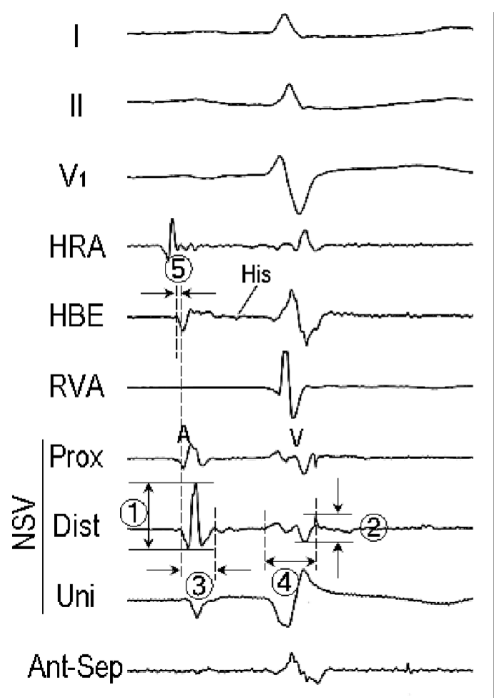

B

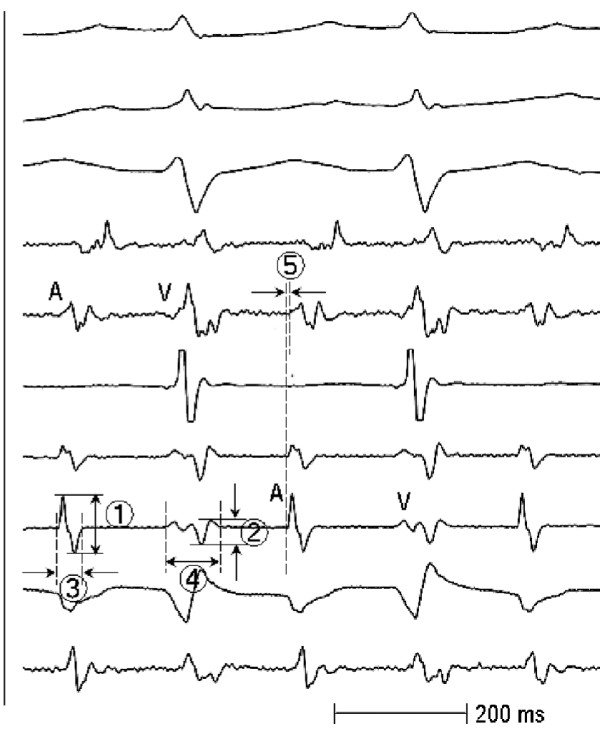

Figure 1. Measurement variables recorded at the non-coronary sinus of Valsalva (NSV) during sinus rhythm (A) and atrial tachycardia originating from near the atrioventricular node (B). The maximum amplitudes of the local atrial (1) and ventricular (2) electrograms recorded at the NSV, the amplitude ratio of the local atrial electrogram to the ventricular electrogram at the NSV recording site, the duration of the local atrial (3) and ventricular (4) electrograms, and the interval from the local atrial activation recorded at the His bundle region to that recorded at the NSV (5) were measured. A, atrial electrogram; Ant-Sep, right atrial anteroseptum; Dist, distal; HBE, His bundle electrogram; HRA, high right atrium; Prox, proximal; RVA, right ventricular apex; Uni, unipolar; V, ventricular electrogram. application from the NSV. Four patients had a focal, reentrant AT originating from near the AVN and His-bundle region, and the remaining 1 had a concealed anteroseptal accessory pathway, 1,2 Two of them were reported as case reports 1,2 All patients had a normal ECG during sinus rhythm, and no structural abnormalities were found by physical examination and echocardiography.

Protocol II: Recording of the NSV Electrograms in 20 Control Subjects For further characterization of the local electrograms recorded from the NSV and clarification of the prevalence of recording a His-bundle potential at the NSV, the NSV electrograms were recorded during sinus rhythm in 20 control subjects (11 women; mean age, $54 \pm 19$ years) after a successful ablation of their original tachycardia to determine the electrogram characteristics; 12 subjects had AVRT caused by a concealed accessory pathway at the left ventricular free wall and 5 had idiopathic premature ventricular contractions originating from the left ventricle. The remaining 3 had focal AT originating from the crista terminalis. Coronary angiography was performed in those 3 subjects because of the concomitance of chest pain, but it resulted in no significant coronary arterial stenosis.

Protocol III: Comparison of the Electrograms Recorded From the Aortic Sinus of Valsalva in 7 Control Subjects For characterization of the local electrograms recorded from the NSV, electrograms from the left and right aortic sinus of Valsalva (LSV and RSV), as well as the NSV, were recorded in another 7 control subjects ( 2 women; mean age, $49 \pm 16$ years) after a successful ablation of their original tachycardia to determine the electrogram characteristics; 5 subjects had AVRT caused by a concealed accessory pathway at the left ventricular free wall, and the remaining 1 had idiopathic premature ventricular contractions originating from the left ventricle.

All subjects participating in protocols II and III had a normal ECG during sinus rhythm, and no structural abnormalities were found by physical examination and echocardiography. Ethical approval was obtained from the hospital ethics committee, and all patients gave informed consent before participation.

\section{Diagnosis and Catheter Ablation in Patients With Supraventricular Tachycardia}

In all 5 patients with PSVT, focal ATs near the His-bundle region $(n=4)$ or an AVRT $(n=1)$ caused by a concealed anteroseptal accessory pathway were precisely diagnosed and ablated successfully as described previously!,2 Briefly, in all 4 patients with AT, the tachycardia was induced and terminated reproducibly by atrial extrastimulation (Figure 1A). The tachycardia was reproducibly induced by atrial pacing or ventricular pacing, with advancement of the atrial electrograms and a V-A-A-V activation sequence. Termination of the tachycardia by ventricular pacing failed, without any advancement of the atrial electrograms. Furthermore, the tachycardia exhibited a "V-A-A" response upon the cessation of pacing after entrainment with ventricular pacing. 10 The intra-atrial activation sequence during the tachycardia differed from that seen during ventricular pacing. The tachycardia could be terminated reproducibly by using a small dose $(2.5-5 \mathrm{mg})$ of adenosine triphosphate without any development of AV nodal conduction block in the 4 patients we tested. The effect of a small dose of adenosine triphosphate on the tachycardia could not be tested in the remaining patient because of the concomitance of bronchial asthma. From the findings described above, we diagnosed those tachycardias as an AT originating from near the AV nodal transitional area1,11 In a patient with a concealed anteroseptal accessory pathway, the earliest retrograde atrial activation during the ventricular pacing was found at the NSV?

In the initial 2 patients, with an AT and a concealed anteroseptal accessory pathway, the RF energy delivery was initially performed at the RA anteroseptum. Because the ablation at that site failed, the NSV was mapped and complete elimination of the AT and accessory pathway could be obtained. In the remaining 3 patients with AT, the local atrial activation during the AT was earlier at the NSV than at the RA anteroseptum and the NSV was the site where the earliest activation during the AT was recorded. Therefore, an RF energy delivery was initially performed at the NSV.

After confirming the location of the ostia of the coronary arteries and identifying the contour of the aortic sinus of Valsalva by using a hand injection of a contrast agent, the earliest atrial activation during the supraventricular tachy- 
Table 1. Results of the Measurements of the Bipolar Electrograms During Normal SR and the Tachycardia in 5 Patients With Paroxysmal SVT

\begin{tabular}{|c|c|c|c|c|c|}
\hline & \multicolumn{2}{|c|}{ Patients with tachycardia $(\mathrm{n}=5)$} & \multirow{2}{*}{$P$ value ${ }^{1}$} & \multirow{2}{*}{$\frac{\text { Control subjects }(\mathrm{n}=20)}{\text { During SR }}$} & \multirow{2}{*}{ P value ${ }^{2}$} \\
\hline & During SR & During tachycardia & & & \\
\hline \multicolumn{6}{|l|}{ Amplitude } \\
\hline $\mathrm{AE}(\mathrm{mV})$ & $0.39 \pm 0.38(0.10-0.47)$ & $0.22 \pm 0.17(0.04-0.48)$ & 0.3 & $0.34 \pm 0.24 *(0.10-1.02)$ & 0.7 \\
\hline Ventricular E (mV) & $0.17 \pm 0.08(0.07-0.29)$ & $0.23 \pm 0.10(0.10-0.35)$ & 0.2 & $0.14 \pm 0.12(0.08-0.29)$ & 0.7 \\
\hline AE/ventricular E & $2.26 \pm 1.43(0.68-3.72)$ & $1.04 \pm 0.73(0.12-2.16)$ & 0.1 & $3.13 \pm 2.47(0.69-11.33)$ & 0.5 \\
\hline $\begin{array}{l}\text { Reduction in the atrial E/ventricular E } \\
\text { during tachycardia (Pts No.; \%) }\end{array}$ & & $4(80 \%)$ & & & \\
\hline Atrial E/ventricular E>1 (Pts No.; \%) & $3(60 \%)$ & $2(40 \%)$ & 0.4 & $17(85 \%)$ & 0.2 \\
\hline \multicolumn{6}{|l|}{ Duration } \\
\hline $\mathrm{AE}(\mathrm{ms})$ & $53.8 \pm 11.0(36-76)$ & $64.5 \pm 16.1(40-85)$ & 0.1 & $60.9 \pm 11.6(36-77)$ & 0.7 \\
\hline Ventricular E (ms) & $84.6 \pm 12.5(74-105)$ & $78.8 \pm 26.7(37-110)$ & 0.5 & $73.9 \pm 17.4(38-105)$ & 0.2 \\
\hline \multicolumn{6}{|l|}{ Interval } \\
\hline $\mathrm{AE}[\mathrm{NSV}]-\mathrm{AE}[\mathrm{His}](\mathrm{ms})$ & $-3.6 \pm 3.5(-3-9)$ & $14.5 \pm 11.5(0-31)$ & 0.2 & $0.67 \pm 2.99(-3-7)$ & 0.08 \\
\hline Recording of the His bundle potential at the NSV (\%) & $1(20 \%)$ & ND & ND & $3(15 \%)$ & ND \\
\hline Distance between the His and NSV $(\mathrm{mm})$ & $7.7 \pm 3.7(3.1-11.4)$ & ND & ND & $9.2 \pm 4.4(3.1-18.2)$ & ND \\
\hline
\end{tabular}

Values are the mean \pm SD.

Distance between the His and NSV was defined as the minimum distance between the tip of the catheter at the NSV and the electrodes of the catheter where the His bundle potential was clearly recorded and measured by multiplane fluoroscopy. In one patient with an anteroseptal accessory pathway, the measurements were performed during ventricular pacing with a cycle length of $600 \mathrm{~ms}$.

${ }^{1} \mathrm{P}$ value comparing the electrograms between those recorded during SR and those during the tachycardia in patients with SVT; ${ }^{2} \mathrm{P}$ value comparing the electrograms between the patients with SVT and the control subjects during SR.

$* \mathrm{P}=0.005$ vs ventricular electrogram.

SR, sinus rhythm; SVT, supraventricular tachycardia; AE, atrial electrogram; E, electrogram; Pts, patients; No., number; NSV, non-coronary sinus of Valsalva; ND, not determined.

cardia was recorded within the NSV in all patients. RF energy delivery within the NSV was performed at the site where the earliest activation was recorded during the tachycardia in the cases of AT or during right ventricular pacing in a case of a concealed accessory pathway. RF energy was delivered at that site using a 7 Fr quadripolar catheter with a $4 \mathrm{~mm}$ distal electrode (Biosense Webster, Diamond Bar, CA, USA) with a setting at a maximum power of $35 \mathrm{~W}$ and a maximum electrode-tissue interface temperature of $55^{\circ} \mathrm{C}$ for 60-120s. However, the power delivered was increased gradually from $5 \mathrm{~W}$.

\section{Mapping at the Aortic Sinus of Valsalva and Analysis of the Electrograms}

The mapping and recording of the local electrograms recorded at the aortic sinus of Valsalva were performed with a 7 Fr quadripolar catheter (Biosense Webster). The NSV electrograms were analyzed quantitatively during normal sinus rhythm and the tachycardia in all patients with tachycardias (Protocol I) and in a total of 27 control subjects during normal sinus rhythm (Protocols II and III). The LSV and RSV electrograms were also analyzed in 7 control subjects during sinus rhythm (Protocol III).

\section{Analysis of the Electrograms Recorded at the Aortic Sinus of Valsalva}

A quantitative analysis of the bipolar electrograms recorded by the mapping catheter was performed post hoc. Measurements of the electrogram amplitude, activation time, and duration were performed by using electronic calipers. Analysis of the electrograms recorded at the aortic sinus of Valsalva consisted of the following measurements (Figure 1): (1) the maximum amplitudes of the local atrial and ventricular electrograms; (2) the amplitude ratio of the local atrial electrogram to ventricular electrogram; (3) the duration of the local atrial and ventricular electrograms; and (4) the interval from the local atrial activation recorded at the His-bundle region to that recorded at the NSV. The electro- gram onset was defined as the first deflection that had a slope greater than $45^{\circ}$ at a recording speed of $100 \mathrm{~mm} / \mathrm{s}^{12}$ An electrogram deflection was defined as a positive or negative deflection that had an amplitude of $\geq 0.05 \mathrm{mV} !^{13}$ The minimum distance between the tip of the mapping catheter at the NSV and the electrode of the catheter where the His-bundle potential was clearly recorded was measured at multiple projections, mainly in the left anterior oblique projection.

\section{Statistical Analysis}

Continuous variables are expressed as the mean \pm standard deviation, and were compared using the Student's t-test. An analysis of variance (ANOVA) was used when comparisons involved $>2$ groups. When group differences were found, a one-way ANOVA was used followed by the Scheffe method to test the significance of the difference among the means in all groups. Categorical variables were compared using a chi-square analysis using the Yate's correction if necessary. A P value of $<0.05$ was considered statistically significant.

\section{Results}

\section{NSV Electrograms in 5 Patients With Tachycardias (Protocol I)}

Both the atrial and ventricular potentials were recorded within the NSV during sinus rhythm and the tachycardia in all patients. During sinus rhythm, the amplitude of the atrial electrogram recorded at the NSV $(0.39 \pm 0.38 \mathrm{mV})$ was greater than that of the ventricular electrogram $(0.17 \pm$ $0.38 \mathrm{mV})$, although there was no significant difference $(\mathrm{P}=$ 0.18 ; Table 1). The amplitude ratio of the atrial electrogram to ventricular electrogram was $2.26 \pm 1.43$, and it occurred in more than 1 in $3(60 \%)$ patients. The mean interval from the onset of the atrial electrogram at the His-bundle region to that at the NSV was $-5.8 \pm 4.1 \mathrm{~ms}$, indicating that the atrial activations at both sites occurred almost simultaneously (Table 1). 
A

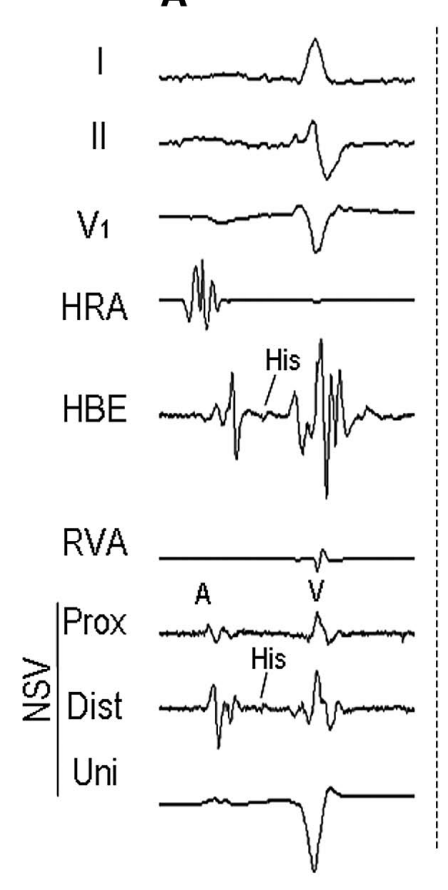

B

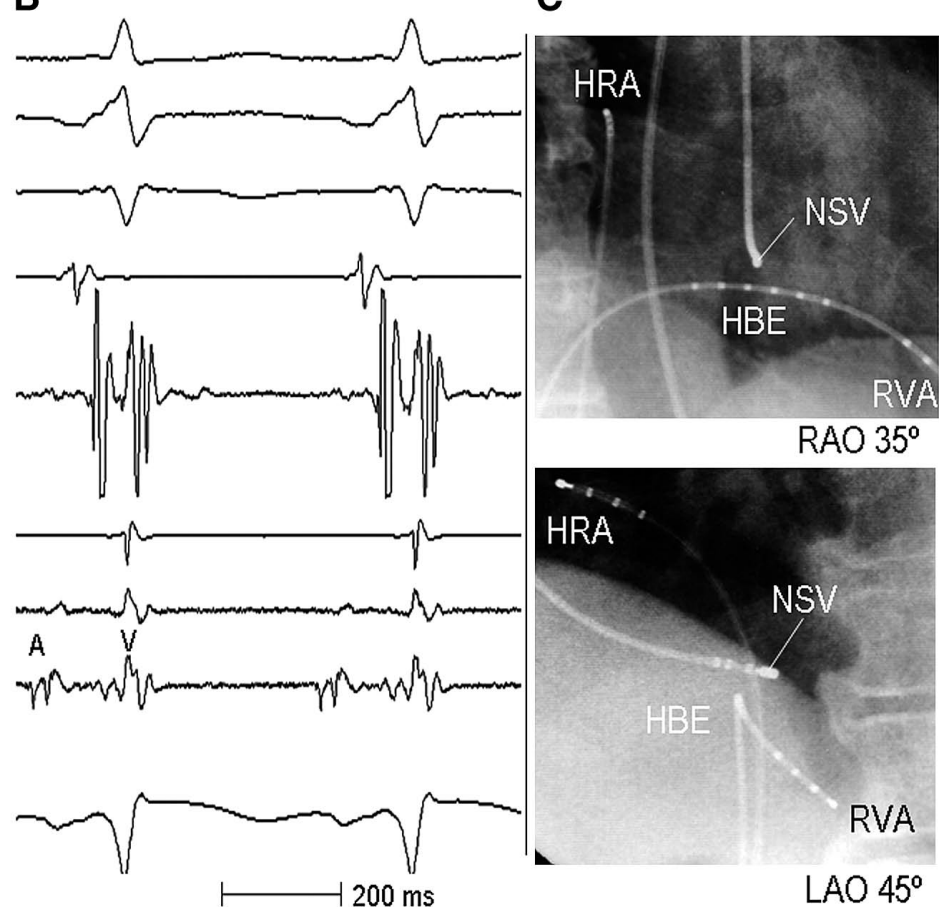

Figure 2. Representative electrograms recorded at the NSV during sinus rhythm (A) and atrial tachycardia (B) in a patient with atrial tachycardia originating from near the atrioventricular node. The radiograms obtained in the right anterior oblique $\left(\mathrm{RAO} 35^{\circ}\right)$ and left anterior oblique (LAO $45^{\circ}$ ) projections show the successful ablation sites $(\mathbf{C})$. A tiny but distinct His bundle deflection (His) was recorded from the distal electrode of the ablation catheter positioned within the NSV. Abbreviations as in Figure 1.

In all patients during the tachycardia, the earliest atrial activation was found within the NSV where the local electrogram during the tachycardia recorded from the distal electrodes of the ablation catheter preceded the onset of the surface $\mathrm{P}$ wave by $44 \pm 9 \mathrm{~ms}$ (range, $38-58$ ). The amplitude of the atrial electrogram became smaller and the amplitude of the ventricular electrogram was greater during the tachycardia than during sinus rhythm, although there was no significant difference (Table 1; Figures 1,2). As a result, the amplitude of the atrial electrogram was comparable with that of the ventricular electrogram $(\mathrm{P}=0.9)$, and the amplitude ratio of the atrial electrogram to ventricular electrogram got smaller during the tachycardia than during sinus rhythm (Table 1). In $4(80 \%)$ patients, it's ratio decreased during the tachycardia.

In $1(20 \%)$ patient, a tiny potential with the same timing as the His-bundle deflection with an amplitude of $0.015 \mathrm{mV}$ was found, but it could not be recognized during the tachycardia (Figure 2). The duration of the atrial and ventricular electrograms did not differ between that obtained during sinus rhythm and that during the tachycardia (Table 1). There was a tendency for a greater increase in the interval from the onset of the atrial electrogram at the NSV to that at the His-bundle region during the tachycardia than during sinus rhythm (Table 1). The mean distance between the recording site within the NSV and the His-bundle potential recording site in the right atrium was $7.7 \pm 3.7 \mathrm{~mm}$.

In a patient with a concealed anteroseptal accessory pathway, catheter ablation was performed during right ventricular pacing. In the initial 3 patients with ATs, RF energy was delivered during the AT. However, in the most recent patient with AT, catheter ablation was performed during sinus rhythm after confirming that the earliest atrial activation during the AT was recorded at the NSV. With incremental atrial pacing after the ablation procedure, the paced cycle length producing the AV nodal Wenckebach block was identical with that before the ablation procedure in all patients. The A-H interval and $\mathrm{H}-\mathrm{V}$ interval after the ablation were $124 \pm 16 \mathrm{~ms}$ and $33 \pm 3 \mathrm{~ms}$, respectively, which did not differ from that after the ablation (A-H interval, 118 \pm $19 \mathrm{~ms}, \mathrm{P}=0.10 ; \mathrm{H}-\mathrm{V}$ interval, $34 \pm 5 \mathrm{~ms} ; \mathrm{P}=0.19)$. No clearly prolonged $\mathrm{A}-\mathrm{H}$ or $\mathrm{H}-\mathrm{V}$ interval was found in any of the patients after the ablation.

All 5 patients were discharged without any medications after the RF ablation at the NSV, and have done well with no tachycardia recurrences during a follow up of $42 \pm 28$ months (range, 9-75 months). No complications occurred during the ablation procedure or follow up. No aortic valve abnormalities or deterioration of the left ventricular function was found by echocardiography during the follow up either.

\section{NSV Electrograms in 20 Control Subjects}

In 20 subjects in whom the local electrogram was recorded within the NSV during sinus rhythm, the amplitude of the atrial electrogram recorded at the NSV was greater than that of the ventricular electrogram $(\mathrm{P}=0.005$; Table 1). The amplitude ratio of the atrial electrogram to the ventricular electrogram was more than 1 in $17(85 \%)$ subjects. No significant difference was found in any of those parameters assessed between those 20 control subjects and the 5 patients with tachycardias (Table 1). The mean distance between the NSV and His-bundle potential recording site at the right anteroseptum was $9.2 \pm 4.4 \mathrm{~mm}$. In $3(15 \%)$ subjects, a tiny 
A
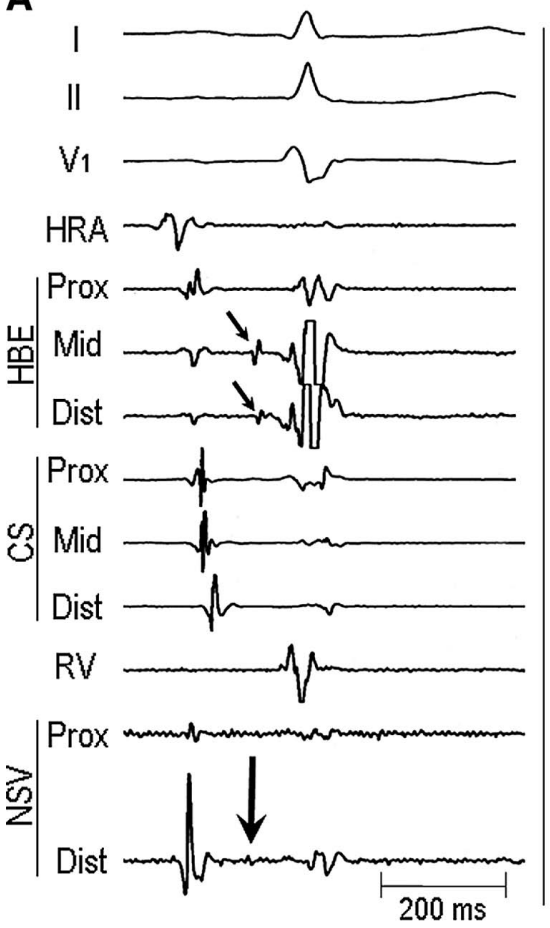

A

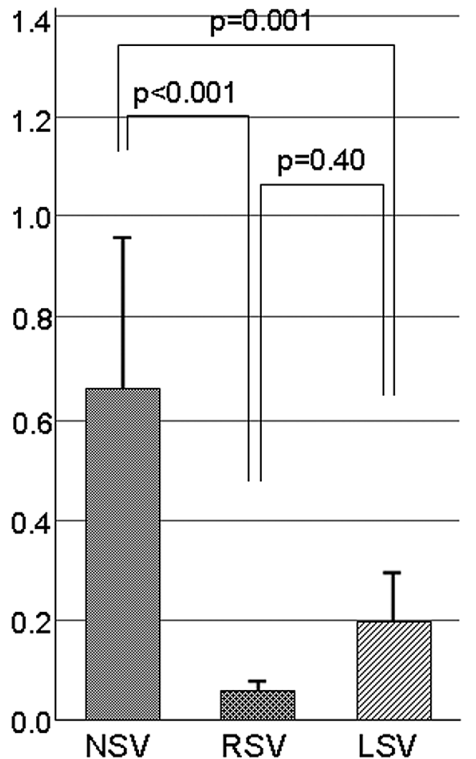

B

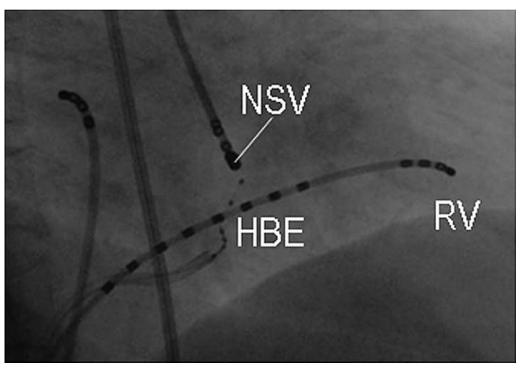

$\mathrm{RAO} 35^{\circ}$

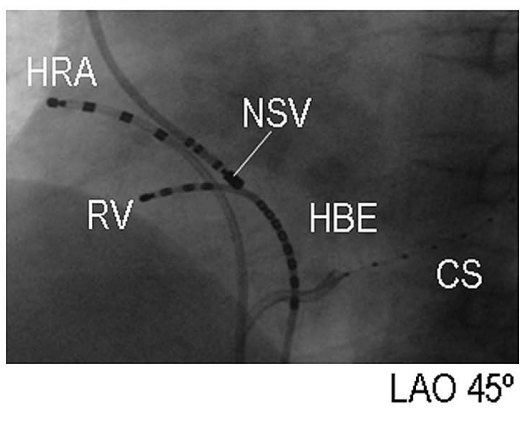

Figure 3. Representative electrograms recorded at the non-coronary sinus of Valsalva (NSV) during sinus rhythm (A) and the radiograms showing the mapping site at the NSV (B) in a control subject. Note that a distinct Hisbundle deflection (large arrow) was recorded with the same timing as that recorded at the His-bundle region (HBE; small arrows). CS, coronary sinus; Mid, middle; RV, right ventricle. Abbreviations as in Figures 1 and 2.
B

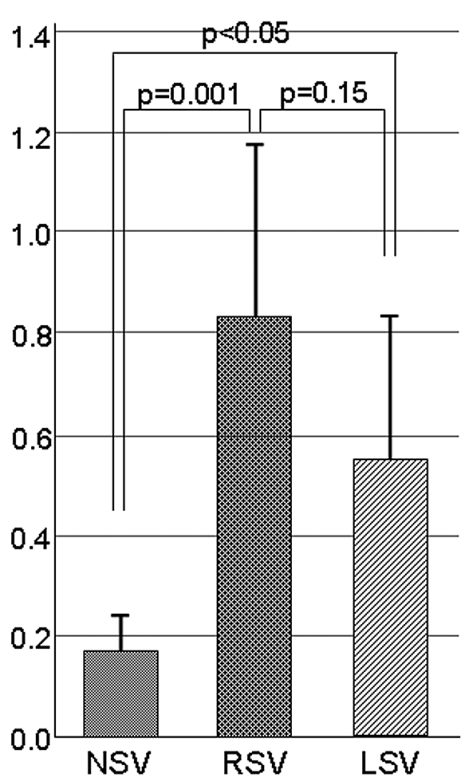

C

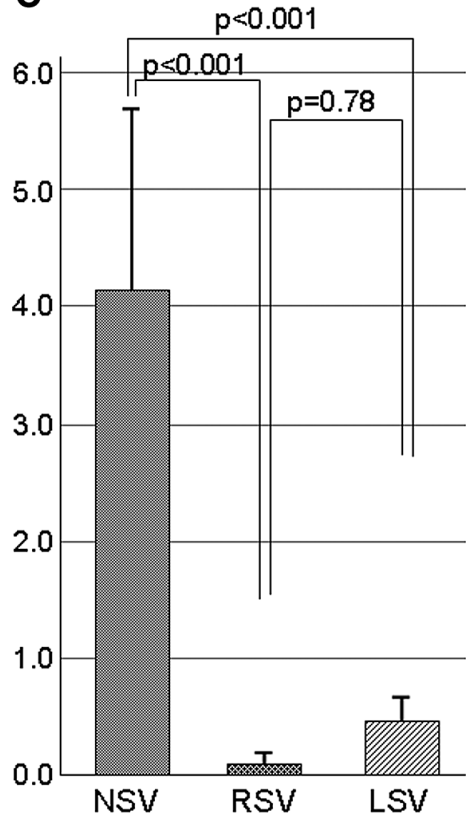

Figure 4. Comparison of the local electrograms recorded at the aortic sinus of Valsalva in 7 control subjects. (A) The amplitude of the atrial electrogram. (B) The amplitude of the ventricular electrogram. (C) The amplitude ratio of the atrial electrogram to the ventricular electrogram. LSV, left sinus of Valsalva; NSV, non-coronary sinus of Valsalva; RSV, right sinus of Valsalva.

but distinct His-bundle deflection with a mean amplitude of $(0.018 \pm 0.006 \mathrm{mV})$ was recorded at the NSV (Figure 3).

\section{Comparison of the Electrograms Recorded at the Aortic Sinuses of Valsalva}

In 7 subjects in whom the electrograms recorded were compared among the 3 aortic sinuses of Valsalva, the amplitude of the atrial electrogram recorded at the NSV was greater than that at the LSV $(\mathrm{P}<0.001)$ or RSV $(\mathrm{P}=0.001$; Figure 4). Inversely, the amplitude of the ventricular electrogram recorded at the NSV was smaller than that at the LSV ( $\mathrm{P}=0.001)$ or RSV ( $\mathrm{P}=0.048$; Figure 4). In all 7 subjects, the amplitude of the atrial electrogram was greater than that of the ventricular electrogram, and the amplitude ratio of the atrial electrogram to the ventricular electrogram was the greatest in the electrograms recorded at the NSV 
Table 2. Comparison of the Local Electrograms Recorded From the Aortic Sinuses of Valsalva in 7 Control Subjects

\begin{tabular}{|c|c|c|c|c|}
\hline & NSV & LSV & RSV & $\mathrm{P}$ value \\
\hline \multicolumn{5}{|l|}{ Duration } \\
\hline $\mathrm{AE}(\mathrm{ms})$ & $62.6 \pm 7.3(54-74)$ & $48.1 \pm 11.3(34-70)$ & $43.9 \pm 15.5(12-56)$ & $<0.05$ \\
\hline Ventricular E (ms) & $80.1 \pm 11.1(70-96)$ & $79.7 \pm 15.1(59-107)$ & $81.3 \pm 17.4(67-116)$ & 0.98 \\
\hline \multicolumn{5}{|l|}{ Interval } \\
\hline $\mathrm{AE}[\mathrm{NSV}]-\mathrm{AE}[\mathrm{His}](\mathrm{ms})$ & $4.9 \pm 6.6(-1-18)$ & $-3.3 \pm 14.2(-23-16)$ & $5.3 \pm 10.1(-9-19)$ & 0.27 \\
\hline Recording of the His bundle potential (Pts. No.; \%) & $1(14 \%)$ & $0(0 \%)$ & $4(57 \%)^{* *}$ & $<0.05$ \\
\hline Distance between the His and NSV (mm) & $8.6 \pm 4.2 *(3.5-15.0)$ & $29.5 \pm 10.1(15.8-43.3)$ & $15.2 \pm 5.5 * * *(7.2-20.3)$ & 0.0004 \\
\hline
\end{tabular}

Values are the mean \pm SD.

Distance between the His and NSV was defined as the minimum distance between the tip of the catheter at the NSV and the electrodes of the catheter where the His bundle potential was clearly recorded and measured by multiplane fluoroscopy. In one patient with an anteroseptal accessory pathway, the measurements were performed during ventricular pacing with a cycle length of $600 \mathrm{~ms}$.

$* \mathrm{P}=0.0005$ vs LSV. ** $\mathrm{P}=0.037$ vs LSV. *** $\mathrm{P}=0.01$ vs LSV.

L (R) SV, left (right) sinus of Valsalva. Other abbreviations see in Table 1.
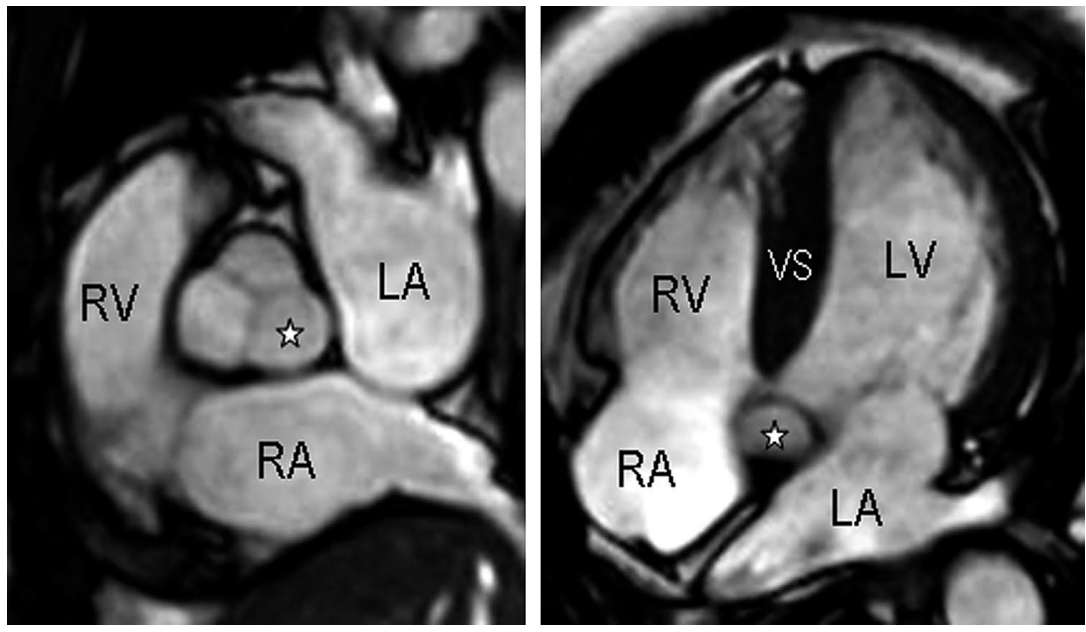

Figure 5. Cross-sectional magnetic resonance image at the level of the cardiac base showing the topographic relationship of the aortic sinus of Valsalva to the surrounding cardiac structures in a control subject. (Left) Short-axis view. (Right) Apical 5 chamber view. The asterisk denotes the non-coronary sinus of Valsalva. $\mathrm{L}(\mathrm{R}) \mathrm{A}$, left (right) atrium; $\mathrm{L}(\mathrm{R}) \mathrm{V}$, left (right) ventricle; VS, ventricular septum.

(both for $\mathrm{P}<0.001$; Figure 4, Table 2). No significant difference was found in those parameters or the interval from the local atrial activation recorded at the His-bundle region between the LSV and RSV (Table 2). A tiny His-bundle deflection was recorded in $1(14 \%)$ subject at the NSV and 4 subjects (57\%) at the RSV. The mean distance from the NSV to the site at the RA anteroseptum where the Hisbundle potential was recorded was $8.6 \pm 4.2 \mathrm{~mm}$, which was shorter than that from the LSV or RSV to the site at the RA anteroseptum (Table 2).

\section{Discussion}

\section{Major Findings}

The results of the quantitative analysis of this study demonstrated the following findings: (1) the local electrogram recorded at the NSV during sinus rhythm had a larger atrial potential amplitude than the ventricle one and the ratio of the atrial amplitude to the ventricular amplitude was often $>1$, which apparently differed from that recorded at the LSV and RSV; and (2) A tiny but distinct His-bundle deflection was recorded at the NSV during sinus rhythm in a total of 5 $(15.6 \%)$ subjects in Protocols I to III of this study. However, in 5 patients in whom the tachycardia could be ablated from the NSV in Protocol I, it could not be confirmed during tachycardia, and a reduction in the amplitude of the atrial electrogram and amplitude ratio of the atrial electrogram to the ventricular electrogram were observed in most patients.

These results might demonstrate that the identification of the catheter position within the NSV among the aortic sinuses of Valsalva is possible with the characteristic findings of the electrograms, and that much attention should be paid during the RF energy delivery at the NSV because of the vicinity of the NSV to the AVN and His-bundle region, and because of the recording of the His-bundle potential within the NSV in some patients. To examine the presence of a His-bundle potential and to watch out for the appearance of any impairment to the AV conduction during an RF energy delivery, performing the RF ablation during sinus rhythm may be recommended.

\section{Anatomical Location of the NSV and its Surroundings and the Electrogram Characteristics of the Aortic Sinuses of Valsalva}

Among the aortic sinuses of Valsalva, the NSV is located most posterior, and is located between the right atrium and left atrium. Thus, it contacts both atria directly (Figure 5 Left). The NSV was also located near the interventricular septum. However, it is above the ventricular myocardial septum, and the membranous septum was positioned between the NSV and ventricular myocardial septum (Figure 5 Right). Those anatomical structures might be responsible for the characteristic electrograms of the NSV, which had a greater amplitude of the atrial electrogram than that of the ventricular electrogram, and the longest duration of the atrial electrogram among the aortic sinuses of Valsalva in this study. In contrast, both the LSV and RSV come in direct contact with the ventricular myocardium. A part of the LSV 
comes in contact with the left atrium, but there is a distance between the greatest portion of the RSV and the atrial myocardium (Figure 5 Left). The anatomical structures of those sinuses of Valsalva might be responsible for our results of a greater amplitude of the ventricular myocardial potential than that of the atrial myocardial potential, and the smallest amplitude of the atrial myocardial potential in the RSV.

The RSV and NSV are close to the anterior and middle portions of the basal portion of the ventricular septum, respectively, ${ }^{14}$ but the LSV was away from the ventricular septum. Therefore, in the present study, a small but distinct His-bundle deflection was recorded at the NSV and RSV in some subjects, but it was not at the LSV in any of the subjects.

\section{Previous Studies}

Several studies including case reports have demonstrated that RF energy applications at the NSV can eliminate AT originating from near the AVN and epicardial anteroseptal accessory pathways, and that the RF energy applications at the LSV and RSV also can eliminate ventricular tachycardia. However, no study has ever tried to determine and compare the characteristics of the electrograms recorded from the aortic sinuses of Valsalva quantitatively or to clarify the prevalence of the recording of a His-bundle deflection from the aortic sinuses of Valsalva. We have clarified those points in the present study.

\section{Clinical Implications}

The results of our data and previous reports ${ }^{1-6}$ demonstrate the following findings: (1) When the earliest atrial activation is found at the RA anteroseptum during mapping in the right atrium and when that site is close to the AVN or His-bundle region, the NSV mapping should always be kept in mind; and (2) When the His-bundle deflection is also recorded at that site, the NSV mapping and a comparison of the timing of the local electrograms at the RA anteroseptum with that of the NSV should be performed.

Right positioning of the mapping/ablation catheter can be confirmed by the characteristics of the electrograms at the aortic sinuses of Valsalva as well as by the fluoroscopic or angiographic views; the amplitude of the local atrial electrogram is more than that of the ventricular electrogram and/or has a longer duration of the atrial electrogram than that of the ventricular electrogram, which might indicate that the catheter is positioned at the NSV. Conversely, a smaller amplitude of the atrial electrogram than the ventricular electrogram and a shorter duration of the atrial electrogram than the ventricular electrogram might indicate that the catheter is positioned at the LSV or RSV.

Fortunately, most reported cases of catheter ablation at the NSV, including our cases of tachycardias originating from near the AVN or His-bundle region, resulted in no impairment of the AV conduction!-6 However, indeed, 1 case that resulted in a complete AV block from the RF energy delivery at the NSV was reported? Therefore, the $\mathrm{RF}$ ablation at the NSV might carry a risk of impairing the AV conduction, and much attention should be paid to the delivery of the RF energy at that site. Whether the Hisbundle potential can be recorded at the NSV should be assessed before the RF energy application at that site is made in order to avoid that complication. However, during the tachycardia, it is difficult to confirm whether there is a His-bundle potential. Greater baseline drift and artifact during the tachycardia than during sinus rhythm or a wider distance between the His-bundle region and the ablation catheter during the tachycardia than during sinus rhythm might be the reasons why the His-bundle potential cannot be recognized during the tachycardia. At any rate, the ablation site at the NSV is indeed close to the AVN and His-bundle position. Therefore, we think that the presence of a His-bundle potential should be assessed during sinus rhythm before the ablation at the NSV.

To avoid the occurrence of impairment of the AV conduction during the RF energy delivery, it is useful to watch out for any prolongation of the PQ, A-H, or H-V intervals. The His potential recorded at the NSV during sinus rhythm could not be found during the tachycardia in the same patient. Therefore, we think that the delivery of the RF energy at the NSV during sinus rhythm is safer than that during the tachycardia. We also recommend that the power of the RF energy should be increased gradually from a low wattage (ie, $5 \mathrm{~W}$ ) in order to avoid any potential complications. To avoid perforating the sinus of Valsalva and damaging the aortic valve ${ }^{14}$ the tip temperature should be maintained at $<55^{\circ} \mathrm{C}$. In this study, the tachycardia could be eliminated using this approach with no complications, indicating that our method might be acceptable as an effective and safe method for ablation at the NSV.

\section{Study Limitations}

First, we compared the electrograms recorded from the aortic sinuses of Valsalva. However, the extent of the catheter movement was relatively limited within each aortic sinus of Valsalva, and the catheter position was confirmed only by fluoroscopy and angiography. Therefore, the detailed location of the catheter within the aortic sinus of Valsalva was unclear, and a detailed mapping and analysis of the electrograms within each aortic sinus of Valsalva could not be performed.

Second, our sample size was small and only 5 patients with tachycardia, which could be ablated from the NSV, were included. Therefore, future studies utilizing a 3-dimensional mapping system for a detailed mapping within the aortic sinus of Valsalva that include a larger number of patients might be needed to determine the characteristics of the electrograms within each aortic sinus of Valsalva and to strengthen our results and idea.

And finally, because of the vicinity of the NSV to the AVN, it might be possible to eliminate AV re-entrant tachycardia (AVNRT) with an RF energy application from the NSV. Our assessment of tachycardias in patients with AT might not have completely denied the possibility of AVNRT. We could not clarify those points in this study.

\section{Acknowledgment}

This study was supported, in part, by a grant from the Gunma Prefecture Government, Japan. We are indebted to Ms Rika Utsugi, Mr Tsutomu Nakajima, other physicians in the Division of Cardiology, and the radiological technologists and medical engineer at Gunma Prefectural Cardiovascular Center for their important contribution to this study.

\section{References}

1. Tada H, Naito S, Miyazaki A, Oshima S, Nogami A, Taniguchi K. Successful catheter ablation of atrial tachycardia originating near the atrioventricular node from the noncoronary sinus of Valsalva. Pacing Clin Electrophysiol 2004; 27: 1440-1443.

2. Tada H, Naito S, Nogami A, Taniguchi K. Successful catheter ablation of an anteroseptal accessory pathway from the noncoronary sinus of Valsalva. J Cardiovasc Electrophysiol 2003; 14: 544-546.

3. Ouyang F, Ma J, Ho SY, Bansch D, Schmidt B, Ernst S, et al. Focal 
atrial tachycardia originating from the non-coronary aortic sinus: Electrophysiological characteristics and catheter ablation. J Am Coll Cardiol 2006; 48: 122-131.

4. Huang H, Wang X, Ouyang F, Antz M. Catheter ablation of anteroseptal accessory pathway in the non-coronary aortic sinus. Europace 2006; 8: $1041-1044$.

5. Yamada T, Huizar J, McElderry H, Kay G. Atrial tachycardia originating from the noncoronary aortic cusp and musculature connection with the atria. Heart Rhythm 2006; 3: 1494-1496.

6. Das S, Neuzil P, Albert CM, D'Avila A, Mansour M, Mela T, et al. Catheter ablation of peri-AV nodal atrial tachycardia from the noncoronary cusp of the aortic valve. J Cardiovasc Electrophysiol 2008; 19: $231-237$.

7. Cueelo C, Huang SK, Wagshal AB, Pires LA, Mttleman RS, Bonavita GJ. Radiofrequency catheter ablation of the atrioventricular junction by a supravalvular noncoronary aortic cusp approach. Pacing Clin Electrophysiol 1994; 17: 1182-1185.

8. Sumitomo N, Tateno S, Nakamura Y, Ushinohama H, Taniguchi K, Ichikawa R, et al. Clinical importance of Koch's triangle size in children: A study using 3-dimensional electroanatomical mapping. Circ J 2007; 71: 1918-1921.

9. Ito S, Tada H, Naito S, Kutsumi Y, Miyamori I, Nogami A, et al. Ran- domized comparison of bipolar vs unipolar plus bipolar recordings during atrioventricular junction ablation: Importance and efficacy of unipolar recording. Circ J 2007; 71: 874-879.

10. Lai LP, Lin JL, Chen TF, Ko WC, Lien WP. Clinical, electrophysiological characteristics, and radiofrequency catheter ablation of atrial tachycardia near the apex of Koch's triangle. Pacing Clin Electrophysiol 1998; 21: 1375-1379.

11. Iesaka Y, Takahashi A, Goya M, Soejima Y, Okamoto Y, Fujiwara H, et al. Adenosine-sensitive atrial reentrant tachycardia originating from the atrioventricular nodal transitional area. J Cardiovasc Electrophysiol 1997; 8: 854-864.

12. Paul T, Moak JP, Morris C, Garison A Jr. Epicardial mapping: How to measure local activation? Pacing Clin Electrophysiol 1990; 13: 285-292.

13. Tada H, Oral H, Greenstein R, Pelosi F, Knight BP, Strickberger SA, et al. Differentiation of atrial and pulmonary vein potential recorded circumferentially within pulmonary veins. $J$ Cardiovasc Electrophysiol 2002; 13: 118-123.

14. Hachiya H, Aonuma K, Yamauchi Y, Igawa M, Nogami A, Iesaka Y. How to diagnose, locate, and ablate coronary cusp ventricular tachycardia. J Cardiovasc Electrophysiol 2002; 13: 551-556. 